\title{
A note on the D-optimality and D-efficiency of nonorthogonal blocked main effects plans
}

\author{
Łukasz Smaga \\ Faculty of Mathematics and Computer Science, Adam Mickiewicz University, \\ Umultowska 87, 61-614 Poznań, Poland, e-mail: ls@amu.edu.pl
}

\begin{abstract}
SUMMARY
This paper considers main effects plans used to study $m$ two-level factors using $n$ runs which are partitioned into $b$ blocks of equal size $k=n / b$. The assumptions are adopted that $n \equiv 2(\bmod 8)$ and $k>2$ is even. Certain designs not having all main effects orthogonal to blocks were shown by Jacroux (2011a) to be D-optimal when $(m-2)(k-2)+2 \leqslant n \leqslant(m-1)(k-2)+2$. Here, we extend that result. For $(m-3)(k-2)+2 \leqslant n<(m-2)(k-2)+2$, the D-optimality of those designs is proved. Moreover, their D-efficiency is shown to be close to one for $2(m+1) \leqslant n<(m-3)(k-2)+2$, indicating their good performance under the criterion of D-optimality.
\end{abstract}

Key words: blocked main effects plan; D-efficiency; D-optimality; Fischer's inequality; Hadamard's inequality; nonorthogonality.

\section{Introduction}

Due to the expensive and time-consuming nature of experiments planned with a complete factorial design, optimal fractional factorial plans are widely applied in diverse fields such as agriculture, industry, and medical research. For example, they are commonly used in industrial research (Bose and Bagchi, 2007; Davies and Hay, 1950; Jacroux, 2009), where an important application is the improvement of manufacturing processes (e.g. chemical and biological processes or formulation of pharmaceutical preparations) and of product quality. In chemical research, optimal fractional factorial plans help to determine the effect of some changes in reaction conditions (e.g. temperature or time of reaction) or methods of manufacture (e.g. fermentation processes). In such research it is usually sufficient to use two-level factorial designs, to examine one change in each of the factors under investigation, e.g. an increase or decrease in the concentration and amounts of 
one or more of the various constituents of the medium. Much of the work on optimal fractional factorial plans is valid in the absence of blocks, although the importance of blocking is emphasized by many researchers (see, for example, Cheng et al., 2004, and the references therein). A practical example of using fractional factorial design with blocks has already been given in section 7 of Davies and Hay (1950), where the effect of various factors on the yield of penicillin is investigated. Recently, interesting results on optimal main effect plans with blocking have been obtained (see references given below). Some of them are extended in the following sections.

In this paper, we consider experimental situations in which $m$ two-level factors are to be studied in $n$ runs, which are partitioned into $b$ blocks of size $k=n / b$, where $k$ is even, and where a main effects only design is to be used. By Jacroux (2011a), we use $d$ to denote a design and the model for analyzing the data under this design is of the form

$$
Y=X_{d} \beta+B_{d} \alpha+\epsilon
$$

where $Y$ is an $n \times 1$ vector of observations, $X_{d}=\left(X_{d_{1}}, \ldots, X_{d_{m}}\right)=\left(x_{d i j}\right)$ is an $n \times m$ design matrix, $\beta=\left(\beta_{1}, \ldots, \beta_{m}\right)^{\prime}$ is the vector of main effects parameters, $B_{d}$ is an $n \times b$ zero-one block design matrix with entries $b_{d i j}=1$ if and only if the $i$ th run occurs in the $j$ th block, $\alpha=\left(\alpha_{1}, \ldots, \alpha_{b}\right)^{\prime}$ is the vector of block parameters and $\epsilon$ is a vector of random error components, which are assumed to be uncorrelated with zero mean and constant variance $\sigma^{2}$. We interchangeably represent a design $d$ by its design matrix $X_{d}$, whose $i$ th row corresponds to run $i$ and whose $j$ th column corresponds to factor $j$, where $x_{d i j}=1,-1$ depending on whether factor $j$ occurs at a high or low level during run $i$. We use $D(n, m, b)$ to denote the class of two-level main effects designs available where $m$ two-level factors occur in $n$ runs arranged in $b$ blocks of size $k=n / b$, and $\bar{D}(n, m, b)$ to denote the subclass of designs within $D(n, m, b)$ having each factor occur at its high and low levels equally often within each block. We also use $D(n, m)$ to denote the entire class of two-level main effects plans having $m$ factors occurring in $n$ runs.

Under the model (1), the least squares estimate for $\beta$ is any solution to the reduced normal equations for main effects given by $M_{d} \hat{\beta}=Q$, where $M_{d}=X_{d}^{\prime} X_{d}-(1 / k) X_{d}^{\prime} B_{d} B_{d}^{\prime} X_{d}, Q=X_{d}^{\prime}\left(I_{n}-(1 / k) B_{d} B_{d}^{\prime}\right) Y$ and $I_{n}$ is the identity matrix of size $n$. The matrix $M_{d}$ is called the information matrix of design $d$.

Among all designs $d$ in the class $D(n, m, b)$, we often wish to choose 
the best one with respect to some criterion. Many criteria are considered in the literature, we shell be interested in one of the most popular, namely the D-optimality criterion. We say a design $d$ is D-optimal in $D(n, m, b)$ if $\operatorname{det}\left(M_{d}\right)$ is maximal. The other optimality criteria are also expressed in terms of the information matrix. Some results concerning such issues are found, among others, Bose and Bagchi (2007), Jacroux (2009, 2011a,b, 2013), Jacroux and Kealy-Dichone (2014, 2015) and Mukerjee et al. (2002). It is worth mentioning, that there are also many other considerations for optimal designs described in the literature (see, for example, Cheng and Tsai, 2009; Cheng et al., 2004; Das and Dey, 2004).

In this paper, we expand the results of the three papers by Jacroux (2011a,b, 2013), which concern the case when $n \equiv 2(\bmod 8)$ and $k$ is even $($ more precisely $k \equiv 2(\bmod 4))$. Jacroux $(2013)$ proved that a design $d_{*} \in \bar{D}(n, m, n / 2)$ having

$$
M_{d_{*}}=(n-2) I_{m}+2 J_{m m},
$$

where $J_{m m}$ is the $m \times m$ matrix of ones, is optimal in $D(n, m, n / 2)$ under the D-optimality criterion as well as most other widely used optimality criteria. When $n \geqslant(m-1)(k-2)+2$ and $k>2$, Jacroux (2011a) showed that a design $d_{*} \in \bar{D}(n, m, b)$ with $M_{d_{*}}$ of the form (2) is D-optimal in $D(n, m, b)$. However, Jacroux (2011b) proved that a D-optimal design does not always belong to $\bar{D}(n, m, b)$, when $n \leqslant(m-1)(k-2)+2$ and $k>2$. In that paper, the following construction is considered.

1. Let $d_{1} \in D((n-2) / 2, m)$ have $X_{d_{1}}$ which consists of $m$ columns from an $(n-2) / 2 \times(n-2) / 2$ Hadamard matrix.

2. Let $d_{2} \in D(n-2, m,(n-2) / 2)$ have ( $\otimes$ denotes the Kronecker product)

$$
X_{d_{2}}=X_{d_{1}} \otimes\left(\begin{array}{r}
1 \\
-1
\end{array}\right)
$$

and where blocks of size two are obtained by taking successive pairs of "foldover" runs in $X_{d_{2}}$.

3. Form $d_{3} \in D(n, m, n / 2)$ by adding a single block to $d_{2}$ consisting of one run of all 1's followed by another run consisting of $m_{1} 1$ 's followed by $n-m_{1}-1$ 's where $m_{1}>0$.

4. Form $d_{4} \in D(n, m, b)$ by combining $k / 2$ successive runs of blocks of size two in $X_{d_{3}}$ to form blocks of size $k$ in $d_{4}$. 
Jacroux (2011b) showed that a design $d_{4}$ obtained via $(3)$ is D-better than the best design in $\bar{D}(n, m, b)$ provided that $n<(m-1)(k-2)+2$, whereas $d_{4}$ is D-equivalent to the best design in $\bar{D}(n, m, b)$ when $n=(m-1)(k-2)+2$. He also noted that for $n \leqslant(m-2)(k-2)+2$, the best design obtainable via (3) has $m_{1}$ which is closest to $\hat{m}_{1}=(m(k-2)-n+2) / 2(k-2)$ where as if $1 \leqslant(m(k-2)-n+2) /(k-2)<2$, the best design will have $m_{1}=1$. From now on, we assume that $d_{4}$ is the best design under the D-optimality criterion obtained via (3), i.e. it has $m_{1}$ which is closest to $\hat{m}_{1}$.

In Jacroux (2011a), the D-optimality of a design $d_{4}$ constructed as in (3) is proved, when $(m-2)(k-2)+2 \leqslant n \leqslant(m-1)(k-2)+2$. Jacroux (2011b) also mentioned that designs $d_{4}$ obtained via (3) seem to be D-optimal when $n<(m-2)(k-2)+2$. In section 2 , we prove that $d_{4}$ is D-optimal when $(m-3)(k-2)+2 \leqslant n<(m-2)(k-2)+2$, extending the result of Jacroux (2011a). For $n<(m-3)(k-2)+2$, it seems to be more difficult to prove the D-optimality of $d_{4}$, but in section 3 we show that the D-efficiency of designs $d_{4}$ is close to one, indicating their very good behavior under the D-optimality criterion and their practical usefulness.

\section{D-optimal designs when}

$$
(m-3)(k-2)+2 \leqslant n<(m-2)(k-2)+2
$$

In this section, we prove the D-optimality of designs $d_{4}$ obtained via (3) for $(m-3)(k-2)+2 \leqslant n<(m-2)(k-2)+2$. In this way, we extend the results of Jacroux (2011a) and partially confirm the conjecture of Jacroux (2011b), as mentioned in the last paragraph of section 1 .

Assume that $n \equiv 2(\bmod 8), k=n / b>2, k$ is even and $(m-3)(k-$ $2)+2 \leqslant n<(m-2)(k-2)+2$. Then, for $(m-3)(k-2)+2<n<$ $(m-2)(k-2)+2, \hat{m}_{1} \in(1,3 / 2)$, so $m_{1}=1$, but for $n=(m-3)(k-2)+2$, we have $\hat{m}_{1}=3 / 2$, and hence $m_{1}$ equals 1 or 2 . By the proof of Theorem 3.1 in Jacroux (2011b), we see that the eigenvalues of the matrix $M_{d_{4}}$ are equal to $z_{d_{4}, 1}=\cdots=z_{d_{4}, m-2}=n-2, z_{d_{4}, m-1}=n+2\left(m_{1}-1\right)-4 m_{1} / k$, $z_{d_{4}, m}=n+2\left(m-m_{1}-1\right)$. Thus for $m_{1}=1$, we have

$$
\operatorname{det}\left(M_{d_{4}}\right)=(n-2)^{m-2}\left(n-\frac{4}{k}\right)(n+2(m-2)) .
$$

Since, for $n=(m-3)(k-2)+2$, we can take $m_{1}=2$, in this case we also 
have

$$
\operatorname{det}\left(M_{d_{4}}\right)=(n-2)^{m-2}\left(n+2-\frac{8}{k}\right)(n+2(m-3)) .
$$

In the following theorem, the D-optimality of the design $d_{4}$ in the class $D(n, m, b)$ is proven.

Theorem 2.1. Let $n \equiv 2(\bmod 8), k=n / b>2$, let $k$ be even and let $d_{4} \in D(n, m, b)$ be constructed as in (3). If $(m-3)(k-2)+2 \leqslant n<$ $(m-2)(k-2)+2$, then $d_{4}$ is D-optimal in the class $D(n, m, b)$.

The proof of Theorem 2.1 is quite long and so we present it in the Appendix.

Remark 2.1. When $n \equiv 2(\bmod 8), k=n / b>2$ and $k$ is even, Theorem 2.1 and Theorem 4.1 of Jacroux (2011a) show that the designs $d_{4}$ obtained as in (3) are D-optimal in $D(n, m, b)$ for $(m-3)(k-2)+2 \leqslant n \leqslant(m-$ $1)(k-2)+2$.

Perhaps the technique presented in the proof of Theorem 2.1 can be used to show the D-optimality of the designs $d_{4}$ for $2(m+1) \leqslant n<(m-$ $3)(k-2)+2$, but this would certainly be quite difficult. However, in the next section, we show the very satisfactory behavior of these designs in terms of D-efficiency.

\section{Highly D-efficient designs for$$
2(m+1) \leqslant n<(m-3)(k-2)+2
$$

In this section, we investigate the efficiency of designs $d_{4}$ constructed as in (3) under the D-optimality criterion, i.e. D-efficiency as defined below. In the cases where a D-optimal design is not known (see Remark 2.1), we indicate that the D-efficiency of $d_{4}$ is very high and close to one.

Following the definition of Bulutoglu and Ryan (2009), the D-efficiency of a design $d$ in the class $D(n, m, b)$ is

$$
\text { D-eff }(d)=\left(\frac{\operatorname{det}\left(M_{d}\right)}{\max _{d_{*} \in D(n, m, b)} \operatorname{det}\left(M_{d_{*}}\right)}\right)^{\frac{1}{m}} .
$$

Unfortunately, $\max _{d_{*} \in D(n, m, b)} \operatorname{det}\left(M_{d_{*}}\right)$ is not known. Thus, we can not calculate the D-efficiency of designs. However, when we know an upper 
bound $U B$ for $\max _{d_{*} \in D(n, m, b)} \operatorname{det}\left(M_{d_{*}}\right)$, we can approach $\mathrm{D}$-eff $(d)$ by a lower bound of the form $\left[\operatorname{det}\left(M_{d}\right) / U B\right]^{1 / m}$. Here we will proceed in this manner. Namely, in the following lemma we prove an upper bound for $\max _{d_{*} \in D(n, m, b)} \operatorname{det}\left(M_{d_{*}}\right)$, which we will use to approximate the D-efficiency of a design $d \in D(n, m, b)$ as described above.

Lemma 3.1. Assume that $n \equiv 2(\bmod 8), k=n / b>2$ and $k$ is even. If $2(m+1) \leqslant n<(m-1)(k-2)+2$ and $d \in D(n, m, b)$, then

$$
\operatorname{det}\left(M_{d}\right) \leqslant(n-2)^{m-l-1}\left(n+2\left\lfloor\frac{n-2}{k-2}\right\rfloor\right)^{l} \cdot\left(n-2+2\left(m-l\left\lfloor\frac{n-2}{k-2}\right\rfloor-l\right)\right),
$$

where $l \in \mathbb{N}$ is such that $l(\lfloor(n-2) /(k-2)\rfloor+1) \leqslant m$ and $(l+1)(\lfloor(n-2) /$ $(k-2)\rfloor+1)>m$, and $\lfloor x\rfloor$ is the integral part of $x$.

Proof. Since $m>(n-2) /(k-2)+1 \geqslant\lfloor(n-2) /(k-2)\rfloor+1$, the number $l$ is correctly defined. Partition $X_{d}$ so that $X_{d}=\left(X_{d_{1}}, \ldots, X_{d_{(l+1)}}\right)$, where $X_{d_{i}}$ is $n \times(\lfloor(n-2) /(k-2)\rfloor+1)$ for $i=1, \ldots, l$, and $X_{d_{(l+1)}}$ is $n \times(m-l(\lfloor(n-$ $2) /(k-2)\rfloor+1)$ ). Fischer's inequality (see, for example, Radhakrishna Rao and Bhaskara Rao, 1998, p. 453) implies

$$
\operatorname{det}\left(M_{d}\right) \leqslant \operatorname{det}\left(M_{d_{1}}\right) \ldots \operatorname{det}\left(M_{d_{(l+1)}}\right),
$$

where $M_{d_{i}}$ is the information matrix of the design $d_{i}$ with the design matrix $X_{d_{i}}, i=1, \ldots, l+1$. Since $d_{i} \in D(n,\lfloor(n-2) /(k-2)\rfloor+1, b)$ for $i=1, \ldots, l$, and $d_{(l+1)} \in D(n, m-l(\lfloor(n-2) /(k-2)\rfloor+1), b)$, Theorem 3.1 in Jacroux (2011a) shows that

$$
\begin{aligned}
& \operatorname{det}\left(M_{d_{i}}\right) \leqslant(n-2)^{\left\lfloor\frac{n-2}{k-2}\right\rfloor}\left(n+2\left\lfloor\frac{n-2}{k-2}\right\rfloor\right), i=1, \ldots, l, \\
& \operatorname{det}\left(M_{d_{(l+1)}}\right) \leqslant(n-2)^{m-l\left(\left\lfloor\frac{n-2}{k-2}\right\rfloor+1\right)-1} \cdot\left(n-2+2\left(m-l\left\lfloor\frac{n-2}{k-2}\right\rfloor-l\right)\right) .
\end{aligned}
$$

Therefore, inequalities (8), (9) and (10) imply (7).

By (6) and Lemma 3.1, we conclude immediately the following corollary, which presents the lower bound for the D-efficiency of a design $d$ in the class $D(n, m, b)$.

Corollary 3.1. Under the assumptions of Lemma 3.1, if $d \in D(n, m, b)$,

$\mathrm{D}-\operatorname{eff}(d) \geqslant\left(\frac{\operatorname{det}\left(M_{d}\right)}{(n-2)^{m-l-1}\left[n+2\left\lfloor\frac{n-2}{k-2}\right\rfloor\right]^{l}\left[n-2+2\left(m-l\left\lfloor\frac{n-2}{k-2}\right\rfloor-l\right)\right]}\right)^{\frac{1}{m}}$. 
Using Corollary 3.1, we can examine the D-efficiency of the designs $d_{4}$ for $2(m+1) \leqslant n<(m-3)(k-2)+2$. The eigenvalues of the matrix $M_{d_{4}}$ are $n-2, n+2\left(m_{1}-1\right)-4 m_{1} / k, n+2\left(m-m_{1}-1\right)$ with multiplicities $m-2,1$ and 1, respectively (see the proof of Theorem 3.1 in Jacroux, 2011b). Hence, by Corollary 3.1 , it follows that

$$
\mathrm{D}-\operatorname{eff}\left(d_{4}\right) \geqslant\left(\frac{(n-2)^{l-1}\left[n+2\left(m_{1}-1\right)-\frac{4 m_{1}}{k}\right]\left(n+2\left(m-m_{1}-1\right)\right)}{\left[n+2\left\lfloor\frac{n-2}{k-2}\right\rfloor\right]^{l}\left[n-2+2\left(m-l\left\lfloor\frac{n-2}{k-2}\right\rfloor-l\right)\right]}\right)^{\frac{1}{m}},
$$

where $l$ is as in Lemma 3.1. We have calculated the values of the right hand side of (11) for many values of $n, m, k$, and we conclude that the lower bound for the D-efficiency of the designs $d_{4}$ is close to one. As an example we present values of this lower bound for some $n, m$ and $k$ in Table 1 . We also observed that the lower bound for the D-efficiency of $d_{4}$ decreases when $m$ increases for fixed $n$ and $k$, which was to be expected. In Table 1 we present only its values for $m=n / 2-1$, the largest number of factors for which we can construct the designs $d_{4}$. So the smallest values of D-efficiency of designs $d_{4}$ are given among all possible values for different $m$. Fortunately, for given $k$, this lower bound increases when $n$ increases, which is also to be expected.

We see that the designs $d_{4}$ have D-efficiency close to one. This indicates that these designs perform very well under the D-optimality criterion and seem to be close to D-optimal. Since the D-efficiency of designs $d_{4}$ is so close to one, it seems that there do not exist designs better than them with respect to the D-optimality criterion, i.e. they are D-optimal. We do not know how to prove this, but we have confirmed it numerically. More precisely, we conducted a numerical search based on the tabu-search algorithm (Harman et al., 2016; Jung and Yum, 1996) to find the D-optimal designs in $D(n, m, b)$ for certain $n, m$ and $k$. We used a modification of the $\mathrm{R}$ code of a tabusearch-based approach for chemical balance weighing designs by Katulska and Smaga (2016) (Domijan, 2012; R Core Team, 2015). It is significant that we did not find D-better designs than $d_{4}$. Summarizing, even if the designs $d_{4}$ obtained via (3) are not D-optimal, which seems to be impossible, they are the best known designs under the criterion of D-optimality, having Defficiency very close to one. Therefore, they can be safely used in practice.

Although we have considered particular designs and a particular optimality criterion, the idea of approaching the efficiency of a design under 
Table 1. Lower bound for the D-efficiency of designs $d_{4}$.

\begin{tabular}{|c|c|c|c|c|c|c|c|c|}
\hline \multicolumn{3}{|c|}{$k=6$} & \multicolumn{3}{|c|}{$k=10$} & \multicolumn{3}{|c|}{$k=14$} \\
\hline$n$ & $m$ & D-eff $\left(d_{4}\right) \geqslant$ & $n$ & $m$ & D-eff $\left(d_{4}\right) \geqslant$ & $n$ & $m$ & D-eff $\left(d_{4}\right) \geqslant$ \\
\hline 18 & 8 & 0.9887 & 50 & 24 & 0.9942 & 42 & 20 & 0.9926 \\
\hline 42 & 20 & 0.9952 & 90 & 44 & 0.9967 & 98 & 48 & 0.9967 \\
\hline 66 & 32 & 0.9969 & 130 & 64 & 0.9977 & 154 & 76 & 0.9978 \\
\hline 90 & 44 & 0.9977 & 170 & 84 & 0.9982 & 210 & 104 & 0.9984 \\
\hline 114 & 56 & 0.9982 & 210 & 104 & 0.9986 & 266 & 132 & 0.9987 \\
\hline
\end{tabular}

a chosen criterion of optimality, as used in this paper is widely applicable. It can be modified for other experimental designs and other optimality criteria, since it is usually quite easy to obtain a lower or upper (depending on the criterion) bound for the value of a criterion (see, for example, Katulska and Smaga, 2016). Studying efficiency in this way, may help to indicate very good or even optimal designs under a given optimality criterion.

\section{Acknowledgement}

The author is grateful to the anonymous referee for the valuable comments and constructive suggestions.

\section{Appendix}

Proof of Theorem 2.1. Theorem 3.1 in Jacroux (2011b) implies that $d_{4}$ is D-better than any design $d \in \bar{D}(n, m, b)$. So let $d \in D(n, m, b)$, but $d \notin$ $\bar{D}(n, m, b)$. Then, $d$ has at least one factor that is non-orthogonal to blocks. So partition the factors in $d$ so that factors $1, \ldots, p$ are not orthogonal to blocks whereas factors $p+1, \ldots, m$ are orthogonal to blocks. We correspondingly partition $X_{d}$ so that $X_{d}=\left(X_{d_{1}}, X_{d_{2}}\right)$, where $X_{d_{1}}$ is $n \times p$ and $X_{d_{2}}$ is $n \times(m-p)$. First, let $p=1$. Since a factor in $X_{d_{1}}$ is non-orthogonal to at least one block, the number $X_{d_{1}}^{\prime} B_{d} B_{d}^{\prime} X_{d_{1}}$ is at least 4 ( $k$ is even) and hence the first diagonal element of $M_{d}$ cannot exceed $n-4 / k$. From Fischer's inequality (see, for example, Radhakrishna Rao and Bhaskara Rao, 1998, p. 453), it follows that

$$
\operatorname{det}\left(M_{d}\right) \leqslant\left(X_{d_{1}}^{\prime} X_{d_{1}}-\frac{1}{k} X_{d_{1}}^{\prime} B_{d} B_{d}^{\prime} X_{d_{1}}\right) \cdot \operatorname{det}\left(X_{d_{2}}^{\prime} X_{d_{2}}-\frac{1}{k} X_{d_{2}}^{\prime} B_{d} B_{d}^{\prime} X_{d_{2}}\right) .
$$


Corollary 3.2 in Jacroux (2013) shows that

$$
\begin{aligned}
& \operatorname{det}\left(X_{d_{2}}^{\prime} X_{d_{2}}-\frac{1}{k} X_{d_{2}}^{\prime} B_{d} B_{d}^{\prime} X_{d_{2}}\right) \leqslant \operatorname{det}\left((n-2) I_{m-1}+2 J_{(m-1)(m-1)}\right)= \\
& =(n-2)^{m-2}(n+2(m-2)) .
\end{aligned}
$$

Since $X_{d_{1}}^{\prime} X_{d_{1}}-(1 / k) X_{d_{1}}^{\prime} B_{d} B_{d}^{\prime} X_{d_{1}} \leqslant n-4 / k$ and by (4), (12) and (13), we have

$$
\operatorname{det}\left(M_{d}\right) \leqslant(n-2)^{m-2}\left(n-\frac{4}{k}\right)(n+2(m-2))=\operatorname{det}\left(M_{d_{4}}\right) .
$$

We now assume that $p \geqslant 2$ and partition $X_{d_{1}}$ so that $X_{d_{1}}=\left(X_{d_{1} 1}, \ldots, X_{d_{1} p}\right)$. Fischer's inequality implies

$$
\begin{aligned}
& \operatorname{det}\left(M_{d}\right) \leqslant \operatorname{det}\left(\left(X_{d_{1} 1}, X_{d_{1} 2}\right)^{\prime}\left(I_{n}-\frac{1}{k} B_{d} B_{d}^{\prime}\right)\left(X_{d_{1} 1}, X_{d_{1} 2}\right)\right) \\
& \cdot \operatorname{det}\left(\left(X_{d_{1} 3}, \ldots, X_{d_{1} p}, X_{d_{2}}\right)^{\prime}\left(I_{n}-\frac{1}{k} B_{d} B_{d}^{\prime}\right)\left(X_{d_{1} 3}, \ldots, X_{d_{1} p}, X_{d_{2}}\right)\right) .
\end{aligned}
$$

Since $n \geqslant((m-2)-1)(k-2)+2$, by Theorem 3.1 in Jacroux (2011a), we conclude that

$$
\operatorname{det}\left(\left(X_{d_{1} 3}, \ldots, X_{d_{1} p}, X_{d_{2}}\right)^{\prime}\left(I_{n}-\frac{1}{k} B_{d} B_{d}^{\prime}\right)\left(X_{d_{1} 3}, \ldots, X_{d_{1} p}, X_{d_{2}}\right)\right)
$$

is less than or equal to $(n-2)^{m-3}(n+2(m-3))$. We first consider the case $n=(m-3)(k-2)+2$. From (5) and (14), it follows that to show that $\operatorname{det}\left(M_{d}\right) \leqslant \operatorname{det}\left(M_{d_{4}}\right)$, it is sufficient to prove that the difference $(n-$ $2)(n+2-8 / k)-\operatorname{det}\left(\left(X_{d_{1} 1}, X_{d_{1} 2}\right)^{\prime}\left(I_{n}-(1 / k) B_{d} B_{d}^{\prime}\right)\left(X_{d_{1} 1}, X_{d_{1} 2}\right)\right)$ is greater than or equal to zero. Denote by $\Sigma_{i j}$ the sum of elements in $X_{d_{1} i}$, which corresponds to the $j$-th block, $i=1,2, j=1, \ldots, b$. Let

$$
L=\left(X_{d_{1} 1}, X_{d_{1} 2}\right)^{\prime}\left(I_{n}-\frac{1}{k} B_{d} B_{d}^{\prime}\right)\left(X_{d_{1} 1}, X_{d_{1} 2}\right)
$$

Then,

$$
L=\left(\begin{array}{cc}
n-\frac{1}{k} \sum_{j=1}^{b} \Sigma_{1 j}^{2} & X_{d_{1} 1}^{\prime} X_{d_{1} 2}-\frac{1}{k} \sum_{j=1}^{b} \Sigma_{1 j} \Sigma_{2 j} \\
X_{d_{1} 2}^{\prime} X_{d_{1} 1}-\frac{1}{k} \sum_{j=1}^{b} \Sigma_{1 j} \Sigma_{2 j} & n-\frac{1}{k} \sum_{j=1}^{b} \Sigma_{2 j}^{2}
\end{array}\right)
$$


Since $k$ is even, $\Sigma_{i j}$ can be equal to $0, \pm 2, \pm 4, \ldots, \pm k$. However, there also exist $j$ and $j_{*}$ such that $\Sigma_{1 j} \neq 0$ and $\Sigma_{2 j_{*}} \neq 0$, because factors in $X_{d_{1} 1}$ and $X_{d_{1} 2}$ are non-orthogonal to at least one block. We have to consider four cases.

Case 1. Let

- $\Sigma_{1 i}^{2} \geqslant 4, \Sigma_{1 j}^{2} \geqslant 4$ for some $i, j, i \neq j$ and $\Sigma_{1 r}^{2} \geqslant 0$ for all $r \neq i, r \neq j$, and $\Sigma_{2 s}^{2} \geqslant 4$ for some $s$ and $\Sigma_{2 t}^{2} \geqslant 0$ for all $t \neq s$,

or

- $\Sigma_{1 i}^{2} \geqslant 4$ for some $i$ and $\Sigma_{1 j}^{2} \geqslant 0$ for all $j \neq i$, and $\Sigma_{2 r}^{2} \geqslant 4, \Sigma_{2 s}^{2} \geqslant 4$ for some $r, s, r \neq s$ and $\Sigma_{2 t}^{2} \geqslant 0$ for all $t \neq r, t \neq s$.

Then, in both cases

$$
\left(n-\frac{1}{k} \sum_{j=1}^{b} \Sigma_{1 j}^{2}\right)\left(n-\frac{1}{k} \sum_{j=1}^{b} \Sigma_{2 j}^{2}\right) \leqslant\left(n-\frac{8}{k}\right)\left(n-\frac{4}{k}\right) .
$$

By (15), (16) and Hadamard's inequality (see, for example, Marshall et al., 2011 , p. 306), we obtain $\operatorname{det}(L) \leqslant(n-8 / k)(n-4 / k)$. Since $2<k<n$, we have

$$
\begin{aligned}
& (n-2)\left(n+2-\frac{8}{k}\right)-\operatorname{det}(L) \geqslant(n-2)\left(n+2-\frac{8}{k}\right)+ \\
& -\left(n-\frac{8}{k}\right)\left(n-\frac{4}{k}\right)=\frac{4(k(n-k)+4(k-2))}{k^{2}}>0 .
\end{aligned}
$$

Case 2. Let

- $\Sigma_{1 i}^{2} \geqslant 4$ for some $i$ and $\Sigma_{1 j}^{2} \geqslant 0$ for all $j \neq i$, and $\Sigma_{2 r}^{2} \geqslant 16$ for some $r$ and $\Sigma_{2 s}^{2} \geqslant 0$ for all $s \neq r$,

or

- $\Sigma_{1 i}^{2} \geqslant 16$ for some $i$ and $\Sigma_{1 j}^{2} \geqslant 0$ for all $j \neq i$, and $\Sigma_{2 r}^{2} \geqslant 4$ for some $r$ and $\Sigma_{2 s}^{2} \geqslant 0$ for all $s \neq r$.

In both of these cases, we have

$$
\left(n-\frac{1}{k} \sum_{j=1}^{b} \Sigma_{1 j}^{2}\right)\left(n-\frac{1}{k} \sum_{j=1}^{b} \Sigma_{2 j}^{2}\right) \leqslant\left(n-\frac{16}{k}\right)\left(n-\frac{4}{k}\right) .
$$


But, $(n-16 / k)(n-4 / k) \leqslant(n-8 / k)(n-4 / k)$, so the same arguments as in Case 1 show that $(n-2)(n+2-8 / k)>\operatorname{det}(L)$.

Case 3. Let $\Sigma_{1 i}^{2}=4, \Sigma_{2 j}^{2}=4$ for some $i, j, i \neq j$, and $\Sigma_{1 r}=0$ for all $r \neq i$, and $\Sigma_{2 s}=0$ for all $s \neq j$. Since $b$ is odd and $k \equiv 2(\bmod 4)$, the number of ones in $X_{d_{1} 1}$ and in $X_{d_{1} 2}$ is even. Thus, Lemma 2.2 in Jacroux et al. (1983) implies $\left|X_{d_{1} 1}^{\prime} X_{d_{1} 2}\right| \geqslant 2$. Therefore, by (15), we obtain

$$
\operatorname{det}(L)=\operatorname{det}\left(\begin{array}{cc}
n-\frac{4}{k} & X_{d_{1} 1}^{\prime} X_{d_{1} 2} \\
X_{d_{1} 2}^{\prime} X_{d_{1} 1} & n-\frac{4}{k}
\end{array}\right) \leqslant\left(n-\frac{4}{k}\right)^{2}-4
$$

Hence

$$
\begin{aligned}
& (n-2)\left(n+2-\frac{8}{k}\right)-\operatorname{det}(L) \geqslant(n-2)\left(n+2-\frac{8}{k}\right)-\left(n-\frac{4}{k}\right)^{2}+4= \\
& =\frac{16(k-1)}{k^{2}}>0 .
\end{aligned}
$$

Case 4. Let $\Sigma_{1 i}^{2}=\Sigma_{2 i}^{2}=4$ for some $i$, and $\Sigma_{1 j}=\Sigma_{2 j}=0$ for all $j \neq i$. Hence, by (15), we have

$$
\operatorname{det}(L)=\left(n-\frac{4}{k}\right)^{2}-\left(X_{d_{1} 1}^{\prime} X_{d_{1} 2} \pm \frac{4}{k}\right)^{2} .
$$

As in Case 3, we conclude that $\left|X_{d_{1} 1}^{\prime} X_{d_{1} 2}\right| \geqslant 2$. Since $k \geqslant 6$,

$$
\left(X_{d_{1} 1}^{\prime} X_{d_{1} 2} \pm \frac{4}{k}\right)^{2} \geqslant\left(2-\frac{4}{k}\right)^{2}
$$

which together with (17) gives

$$
\operatorname{det}(L) \leqslant\left(n-\frac{4}{k}\right)^{2}-\left(2-\frac{4}{k}\right)^{2}=(n-2)\left(n+2-\frac{8}{k}\right) .
$$

Thus, we have proved that $\operatorname{det}\left(M_{d_{4}}\right) \geqslant \operatorname{det}\left(M_{d}\right)$ for arbitrary $d \in D(n, m, b)$ when $n=(m-3)(k-2)+2$. Observe that

$$
\begin{aligned}
& \left(n-\frac{4}{k}\right)(n+2(m-2))-\left(n+2-\frac{8}{k}\right)(n+2(m-3)) \\
& =4 k^{-1}(n-((m-3)(k-2)+2)) .
\end{aligned}
$$


So from (4), for $(m-3)(k-2)+2<n<(m-2)(k-2)+2$, it follows that

$$
\operatorname{det}\left(M_{d_{4}}\right)>(n-2)^{m-2}\left(n+2-\frac{8}{k}\right)(n+2(m-3)) \geqslant \operatorname{det}\left(M_{d}\right),
$$

by the above considerations. This implies that the design $d_{4}$ is D-optimal in the class $D(n, m, b)$.

\section{REFERENCES}

Bose M., Bagchi S. (2007): Optimal main effect plans in blocks of small size. Statistics \& Probability Letters 77: 142-147.

Bulutoglu D.A., Ryan K.J. (2009): D-optimal and near D-optimal $2^{k}$ fractional factorial designs of resolution $V$. Journal of Statistical Planning and Inference 139: 16-22.

Cheng C.S., Tsai P.W. (2009): Optimal two-level regular fractional factorial block and split-plot designs. Biometrika 96: 83-93.

Cheng S.W., Li W., Ye K.Q. (2004): Blocked non-regular two-level factorial designs. Technometrics 46: 269-279.

Das A., Dey A. (2004): Optimal main effect plans with non-orthogonal blocks. Sankhyā 66: $378-384$.

Davies O.L., Hay W.A. (1950): Construction and uses of fractional factorial designs in industrial research. Biometrics 6: 233-249.

Domijan K. (2012): tabuSearch: R based tabu search algorithm. R package version 1.1. http://CRAN.R-project.org/package=tabuSearch

Harman R., Bachratá A., Filová L. (2016): Construction of efficient experimental designs under multiple resource constraints. Applied Stochastic Models in Business and Industry 32: 3-17.

Jacroux M. (2009): Blocking in two-level non-regular fractional factorial designs. Journal of Statistical Planning and Inference 139: 1215-1220.

Jacroux M. (2011a): On the D-optimality of orthogonal and nonorthogonal blocked main effects plans. Statistics \& Probability Letters 81: 116-120.

Jacroux M. (2011b): On the D-optimality of nonorthogonal blocked main effects plans. Sankhyā 73: 62-69.

Jacroux M. (2013): Optimal blocked main effect plans. Statistics 47: 1022-1029.

Jacroux M., Kealy-Dichone B. (2014): On the E-optimality of blocked main effects plans when $n \equiv 3(\bmod 4)$. Statistics \& Probability Letters 87: 143-148.

Jacroux M., Kealy-Dichone B. (2015): On the type I optimality of blocked 2-level main effects plans having blocks of different sizes. Statistics \& Probability Letters 98: $39-43$. 
Jacroux M., Wong C.S., Masaro J. (1983): On the optimality of chemical balance weighing designs. Journal of Statistical Planning and Inference 8: 231-240.

Jung J.S., Yum B.J. (1996): Construction of exact D-optimal designs by tabu search. Computational Statistics \& Data Analysis 21: 181-191.

Katulska K., Smaga Ł. (2016): D-optimal and highly D-efficient designs with nonnegatively correlated observations. Kybernetika 52: 575-588.

Marshall A.W., Olkin I., Arnold B.C. (2011): Inequalities: Theory of Majorization and Its Applications. Second Edition, Springer Series in Statistics, New York.

Mukerjee R., Dey A., Chatterjee K. (2002): Optimal main effect plans with nonorthogonal blocking. Biometrika 89: 225-229.

Radhakrishna Rao C., Bhaskara Rao M. (1998): Matrix Algebra and Its Applications to Statistics and Econometrics. World Scientific, Singapore.

R Core Team (2015): R: A language and environment for statistical computing. R Foundation for Statistical Computing, Vienna, Austria. http://www.R-project. org/ 